\title{
LIMITES ET POTENTIALITÉS DU SYSTÈME MÉDIATIQUE POUR L'EXERCICE DE LA CITOYENNETÉ
}

\begin{abstract}
Séverine De Proost ${ }^{1}$
À l'heure où les instances de socialisation conventionnelle (Famille, École, Église, Armée...) sont dénoncées, jusque dans les médias euxmêmes, comme en perte de puissance du point de vue de l'intégration sociale, le système médiatique dans son ensemble ${ }^{2}$ apparaît de plus en plus comme un nouvel -et relativement puissant- agent de socialisation. Nombreuses sont les recherches en sciences de la communication ayant montré comment les médias favorisent de multiples apprentissages. Ces derniers ne se limitent cependant pas à l'assimilation de valeurs et d'éléments de connaissance de type strictement cognitif; les ressources de sens et de savoir mises à disposition des individus interviennent également dans les processus de structuration des affects et d'élaboration des jugements moraux et esthétiques ${ }^{3}$. Il semble dès lors
\end{abstract}

1 Aspirante au Fonds National de la Recherche Scientifique (FNRS). Groupe d'Étude Médias-TIC de l'Université Libre de Bruxelles

2 Nous visons ici le complexe constitué tant des médias de diffusion (dits aussi "médias de masse") que des médias de communication (dits aussi "technologies de l'information et de la communication" ou "TIC").

3 Pour la télévision, nous pensons en particulier aux travaux de D. PASQUIER:

Recherches en communication, $\mathrm{n}^{\circ} 23$ (2005). 
établi que l'étude théorique de la socialisation par les médias ne peut se contenter d'une socialisation entendue dans un sens restreint, soit comme l'inculcation unilatérale des valeurs de la société dans laquelle l'individu survenant (en particulier l'enfant) est voué à s'incorporer'.

Toutefois, l'inclination récente de la recherche en communication à s'appuyer sur un concept "élargi" de socialisation a de quoi rendre perplexe le scientifique préoccupé par la question des "effets" des médias : c'est qu'en se fondant sur une vaste gamme d'apprentissages réalisés à partir des médias (que nous ne contestons évidemment pas), la sociologie en est, bien souvent, venue à mettre en abyme l'idée même d'un quelconque "effet", pour se focaliser sur l'impact ponctuel des messages médiatiques au sein de contextes de sociabilité précis (tels que la famille, l'école... $)^{2}$. Comment, dans un tel cadre heuristique,

“"Chère Hélène ». Les usages sociaux des séries collège”, Réseaux, n 70, 1995, pp. 9-39; La culture des sentiments. L'expérience télévisuelle des adolescents, Paris, Éditions de la Maison des Sciences de l'Homme, coll. "Ethnologie de la France", 1999. Nous signalons également les études suivantes : T. LIEBES, "Seraisje belle, serais-je riche? Images culturelles de la réussite chez les adolescentes", Réseaux, n 98, 1999, pp. 191-215 ; F. BERTHOLET, "Les séries télévisées, une autre manière d'apprendre à vivre ensemble", in G. BAJOIT, F. DIGNEFFE, J.-M. JASPARD, Q. NOLLET DE BRAUWERE (éd.), Jeunesse et Société. La socialisation des jeunes dans un monde en mutation, Bruxelles, De Boeck Université, 2000, pp. 375-383.

1 Comme l'entendait, en son temps, Émile Durkheim, lequel n'hésitait pas à identifier socialisation et éducation: "L'éducation est l'action exercée par les générations adultes sur celles qui ne sont pas encore mûres pour la vie sociale. Elle a pour objet de susciter et de développer chez l'enfant un certain nombre d'états physiques, intellectuels et moraux que réclament de lui et la société politique dans son ensemble et le milieu spécial auquel il est particulièrement destiné (...). Il résulte de la définition qui précède que l'éducation consiste en une socialisation méthodique de la jeune génération" (Éducation et sociologie (1922), Paris, P.U.F., coll. "Quadrige", 1999, p. 51).

2 Il s'agit là d'une prémisse méthodologique inhérente aux recherches qui se fondent sur les derniers raffinements de la pensée sociologique, notamment d'orientation interactionniste, en matière de socialisation (que, rappelons-le, nous ne contestons pas ici en tant que tels). Ainsi, pour le sociologue Claude Dubar, la socialisation renvoie à "un processus de construction, déconstruction et reconstruction d'identités liées aux diverses sphères d'activité (...) que chacun rencontre au cours de sa vie et dont il doit apprendre à devenir acteur" (La socialisation, Paris, Armand Colin, coll. "U - Série Sociologie", 2000, p. 10). Dès lors, si toute situation sociale -toute "sphère d'activité"- devient, par définition, support d'un processus de socialisation, qui lui-même s'opère à partir de diverses identités construites lors de processus de socialisation antérieurs, il devient très difficile d'isoler un quelconque effet propre 
rendre compte de la "médiatisation englobante de nos sociétés" ? Cette difficulté méthodologique, qui n'est pas non plus sans enjeux éthiques, fait écho au modus vivendi autour duquel se retrouvent, depuis une vingtaine d'années, sous l'influence des "Cultural Studies" anglosaxonnes, tenants du paradigme "critique" (qui défendent l'idée d'effets puissants des médias), d'une part, et tenants du paradigme "pluraliste" (qui défendent l'idée d'effets limités), d'autre part ${ }^{1}$. Les uns et les autres s'accordent désormais quant au fait -certes incontestable-que la communication médiatique n'est pas sans médiations et que différentes lectures peuvent être faites d'un même texte en fonction de l'identité sociale et des ressources culturelles de l'individu. D'où la propension actuelle de la recherche à étudier principalement la rencontre des textes médiatiques avec leurs usagers, i.e. leur réception, ainsi que le contexte de celle-ci ${ }^{2}$. Cette focalisation sur le moment de la réception conduit à la difficulté, déjà soulignée, de tirer des conclusions valant au-delà de la seule situation étudiée, et, en conséquence, à l'impossibilité, quasi naturelle de postuler un impact (puissant ou non). Or, pour les professionnels des médias, praticiens (journalistes ou gestionnaires d'un support médiatique) ou théoriciens (chercheurs), il semble difficile de se départir de l'intuition qu'il existe bel et bien quelque chose comme un "effet".

C'est à cette intuition que nous entendons faire droit ici, en traitant de ce qui constitue, à notre sens, l'une des dimensions fondamentales de la socialisation par les médias : la façon dont ils contribuent à l'élaboration du sens commun. Notre hypothèse est la suivante : bien qu'une large frange de l'étude scientifique des médias -en particulier les études de réception- exclue actuellement la possibilité d'un impact global, il y

aux médias au sein de cette suite de processus.

1 Sur la convergence de ces positions traditionnellement antagonistes, nous renvoyons aux articles suivants: J. CURRAN, "La décennie des révisions. La recherche en communication de masse des années 80", trad., Hermès, n 11/12 (“À la recherche du public. Réception, télévision, médias”), CNRS, 1993, pp. 47-74 ; K. C. SCHRØDER, "Convergence of antagonist traditions? The case of audience research", European Journal of Communication, vol. 2, $\mathrm{n}^{\circ} 1,1987, \mathrm{pp} .7-31$.

2 Pour une généalogie des études de réception, accompagnée d'une critique méthodologique, nous conseillons la lecture de l'article de D. DAYAN, "Les mystères de la réception", Le Débat, n 74, 1992, pp. 146-162. Pour une critique davantage "éthique" : B. LE GRIGNOU, "L' 'ubac' des études de réception de la télévision ", Recherches en communication, $\mathrm{n}^{\circ} 3$, 1995, pp. 173-187. Voir aussi le dossier thématique édité par la revue Réseaux: "Les théories de la réception", n 68, novembre / décembre 1994. 
a bien quelque chose comme un "effet des médias" qui s'exerce, et ce via leur contribution à l'élaboration du sens commun. Autrement dit : le système médiatique interviendrait dans la constitution de l'arrièreplan sémantique en regard duquel est interprété son propre discours. Afin d'étayer cette hypothèse, nous nous appuierons ci-dessous sur le schéma herméneutique de formation du sens commun, élaboré, dès la fin du XIX ${ }^{\mathrm{e}}$ siècle, par le philosophe allemand Wilhelm Dilthey (18331911) ${ }^{1}$. Ce schéma, s'il est vieux de deux siècles, n'en reste pas moins pertinent, selon nous, pour comprendre comment, dans notre société fortement médiatisée, le sens commun ne résulte plus seulement d'un processus de formation autonome, mais en grande partie d'une normativité sélective, propre au système médiatique dans son ensemble (dont les critères de sélection restent d'ailleurs soustraits à tout débat public).

Avant tout : que convient-il d'entendre par "sens commun" ? Ce n'est pas le lieu, ici, de nous attarder sur l'histoire philosophique de cette notion. Néanmoins, il nous semble opportun d'en évoquer certains jalons, afin de montrer en quoi consiste l'apport -et donc l'intérêt- de la théorie de Dilthey. La genèse du concept de sens commun s'enracine dans la philosophie antique. Pour Aristote (IVe siècle A.C.N.), le sens commun se rapporte à la perception des "faits bruts", rendue possible par

[ce] sens central qui aurait pour fonction de coordonner les sensations propres à chaque sens spécial, en les rapportant à un même objet et par là de nous en donner la perception².

Les "sensibles communs" renvoient donc, dans la tradition aristotélicienne, à ce qui est ressenti du fait même que nos cinq sens sont simultanément actifs; par conséquent, le sens commun est d'abord historiquement conçu comme relevant de l'ordre du sensible, de l'expérience empirique individuelle. Au XVIII' siècle, l'École écossaise, et en particulier Thomas Reid (1710-1796), développe une philosophie

1 En particulier dans son ouvrage de 1910, Der Aufbau der geschichtlichten Welt in den Geisteswissenschaften, traduit en français (présenté et annoté) par S. MESURE sous le titre : L'édification du monde historique dans les sciences de l'esprit, Paris, Éditions du Cerf, 1988. Précisons d'emblée que Dilthey ne parle pas de "sens commun" mais plutôt d" "expérience générale de la vie".

2 A. LALANDE, Vocabulaire technique et critique de la philosophie, Paris, P.U.F., 1991, p. 970. 
fondée sur la "certitude du sens commun"1, insistant particulièrement sur l'aspect essentiellement intelligible, et non seulement sensible, de la notion. Le sens commun renvoie dès ce moment

[aux] notions communes à tous les hommes, [aux] principes évidents par eux-mêmes, [aux] jugements primitifs et spontanés qui contiennent les motifs de tous les autres².

Ainsi défini, le concept peut sembler assez proche de ce que l'on aurait, aujourd'hui, intuitivement tendance à désigner par "le sens commun": un ensemble de "notions communes", le "bon sens" en quelque sorte. Toutefois, dans l'optique de Reid, il s'agit d'une faculté qui, en dépit d'être comprise comme une faculté de l'esprit, n'en reste pas moins "immuable", figée au travers des lieux et des époques. Une telle conception s'avère ici inopérante, dans la mesure où, en interdisant de rendre compte de la formation et de la transformation, soit de la genèse, du sens commun (celui-ci étant "donné" une fois pour toutes à tous les hommes), elle ne permet, logiquement, pas non plus d'élucider le rôle que jouent potentiellement les médias dans un tel processus.

Il convient en effet de faire la part entre un "sens commun" essentiellement perçu comme une faculté, figée, de l'esprit, comme "instrument judicatoire" 4 , et un "sens commun" équivalent à un ensemble d'idées "reçues" ("reçues", et donc, aussi, préalablement formées, assi-

1 Celui-ci est conçu par REID comme "[le] fond immuable de l'esprit, sa nature essentielle, dont la raison elle-même n'est que le développement réfléchi et formulé" (A. LALANDE, op.cit., p. 971). C'est nous qui soulignons.

2 Loc. cit. A. Lalande ajoute: "Bien que l'expression sens commun ait deux origines certainement indépendantes et deux sens tout à fait distincts qui en dérivent respectivement, il s'est établi de l'un à l'autre certaines communications. M. Van Biéma nous signale notamment ce texte de Leibniz : 'Ces idées, qu' on dit venir de plus d'un sens, comme celles de l'espace, figure, mouvement, nous sont plutôt venues du sens commun, c'est-à-dire de l'esprit même, car ce sont des idées de l'Entendement pur, mais qui ont du rapport à l'extérieur et que les sens font apercevoir' (Nouveaux Essais, livre II, chap.V)". Sur la postérité de la pensée de Reid, nous renvoyons à l'article de synthèse suivant: E. H. MADDEN, "Common sense school", in E. CRAIG (éd.), Routlegde Encyclopedia of Philosophy, vol. 2, Londres, Routledge, 1998, pp. 446-448. Sur la destinée de la notion de sens commun : S. A. GRAVE, "Common sense", in P. EDWARDS, The Encyclopedia of Philosophy, vol. 2, New York, Mac Millan, 1967, pp. 155-160.

3 Au double sens de ce qui concerne tout un chacun et de ce qui -par suite- est ordinaire.

4 J. LACHELIER, “Sur le Sens commun”, in A. LALANDE, op.cit., p. 970. 
milées, transformées et véhiculées). Soit entre, d'une part, un ensemble de vérités absolument démontrées et intangibles, sur lesquelles on peut tout fonder, y compris la philosophie ${ }^{1}$, et, d'autre part, la doxa ${ }^{2}$, c'està-dire :

l'ensemble des opinions si généralement admises, à une époque et dans un milieu donnés, que les opinions contraires apparaissent comme des aberrations individuelles, qu'il serait inutile de réfuter sérieusement et dont il vaut mieux se moquer, si elles sont légères, ou qu'il faut soigner, si elles deviennent graves $^{3}$.

Dans le contexte qui nous préoccupe, l'intérêt de la conception de Dilthey réside précisément dans le fait qu'elle autorise à dépasser la conception "figée" du sens commun, en considérant que les notions partagées sont intersubjectivement stabilisées (et non données une fois pour toutes). Ce que Dilthey nomme "expérience générale de la vie" renvoie au savoir inhérent à l'expérience de la vie sociale, c'est-àdire un corpus de maximes, de sentences, de dictons, de proverbes..., autant de topoï qui forment une sorte de résumé de ce que les hommes ayant vécu et vivant dans une société donnée ont pu accumuler comme "leçons de vie" assez pertinentes pour être retenues par chacun.

Maintenant, comment s'édifie le "sens commun" chez Dilthey ${ }^{4}$ et comment les médias peuvent-ils interférer dans ce processus ? Dilthey

1 Il s'agit là de la conception défendue par G. E. Moore dans son article "A defence of Common Sense” (1925), dans lequel l'auteur tente de hisser le sens commun au statut de philosophie fondamentale et irrécusable. Cette conception sera discutée par Wittgenstein, lequel ramènera philosophie et sens commun au statut de "jeux de langages"; dans cette optique, le sens commun ne peut prétendre à l'absoluité d'un savoir dogmatique. Sur le débat théorique Moore versus Wittgenstein, ainsi que sur les liens équivoques entre philosophie, en particulier du langage, et doxa, nous renvoyons à : G. HOTTOIS, Du sens commun à la société de communication. Éudes de philosophie du langage. Moore, Wittgenstein, Wisdom, Heidegger, Perelman, Appel, Paris, Libraire Philosophique J. Vrin, coll. "Problèmes et controverses", 1989.

2 La distinction est celle-ci : " $1{ }^{\circ}$ les propositions admises en fait par nos contemporains, propositions d'où tout philosophe qui veut exprimer et communiquer sa pensée est nécessairement obligé de partir ; - $2^{\circ}$ et surtout, le mouvement d'assimilation par lequel de nouvelles propositions obtiennent successivement, en vertu de leur valeur intrinsèque, sans séduction, ni contrainte, l'assentiment de tous les hommes qui peuvent les comprendre." (A. LALANDE, op.cit., p. 972).

3 A. LALANDE, op.cit., p. 972.

4 Précisons que, comme l'indique le titre de l'ouvrage L'édification..., l'ambition 
considère que cet arrière-plan sémantique partagé s'élabore à partir du "monde de la vie (Lebenswelt), c'est-à-dire à partir de l'expérience vécue individuelle. Schématiquement, on peut distinguer cinq grands "moments" de cette élaboration :

- première étape : la "relation vitale" (Lebensbezug), i.e., pour Dilthey : la manière dont un événement affecte l'individu (la sensation pure);

- deuxième étape : 1" "expérience vécue" (Erlebnis) : l'individu ressaisit les "relations vitales" et les "classe" sur le critère du sensible (les expériences qui se ressemblent et celles qui ne se ressemblent pas); c'est la construction du vécu dans le temps et dans l'espace ;

- troisième étape : la "totalité de la vie" (Lebenszusammenhang): chaque individu élabore, à partir de son "expérience vécue", une narration, un récit de vie, qui parachève la construction de son identité :

D'un point de vue fonctionnel, la narration permet l'intégration des expériences vécues hétérogènes dans l'unité temporelle d'un récit stabilisant la diversité du vécu dans une totalité signifiante $^{2}$;

- quatrième étape : "la totalité collective de la vie" ou l'"expérience de la communauté" (Gemeinsamkeit) : les différents récits biographiques sont confrontés dans l'espace public, de sorte à constituer, par généralisation, des histoires "typiques";

première de Dilthey n'est pas de formaliser le processus d'élaboration du sens commun; ce qui amène Dilthey à s'intéresser à cette question, c'est sa volonté de trouver une méthode spécifique pour les sciences de l'esprit-historiques ou humaines (s'il reste d'ailleurs connu aujourd'hui, c'est principalement pour l'opposition qu'il établit entre expliquer et comprendre). Selon Dilthey, les sciences de l'esprit ont une genèse "naturelle" (non artificielle, contrairement aux sciences de la nature) : il existe une sorte de "savoir social spontané" qui sert de "tremplin" naturel aux sciences de l'esprit et sur lequel elles s'appuient ; cela veut dire que le simple fait d'être socialisé constitue un savoir de base (qui sera ensuite élaboré de manière scientifique par les sciences de l'esprit). La question devient alors : comment s'élabore ce "tremplin" ?

1 Ces "moments" ne se situent pas, pour Dilthey, dans une succession temporelle, mais sont simultanés; nous ne les ordonnons, par conséquent, que dans le but d'éclaircir l'exposé. Précisons encore que notre reconstruction de ce processus se fonde notamment sur la lecture de : N. ZACCAÏ-REYNERS, Le Monde de la Vie, vol. 1 : Dilthey et Husserl, Paris, Éditions du Cerf, coll. "Humanités", 1995, pp. 1377.

2 N. ZACCAÏ-REYNERS, Le Monde de la vie, vol. 3 : Après le tournant sémiotique, Paris, Éditions du Cerf, coll. "Humanités", 1996, p. 60. 
- cinquième étape : l'interprétation des histoires typiques ${ }^{1}$ donne lieu au passage aux "morales de l'histoire", qui se stabilisent culturellement sous la forme du sens commun ou de l' "expérience générale de la vie".

Ainsi qu'on le retrouvera dans de nombreux courants sociologiques ultérieurs (notamment d'inspiration constructiviste) ${ }^{2}$, il y a, chez Dilthey, présupposition réciproque entre les expériences singulières et l'expérience générale : tandis que les expériences singulières ne peuvent se comprendre, s'interpréter elles-mêmes qu'à l'aide des ressources de sens disponibles dans le corpus objectif et normatif qu'est le sens commun, l'élaboration de ce sens commun s'alimente elle-même des apports provenant des expériences singulières.

Comment les contraintes inhérentes au système, médiatique en l'occurrence, qui a priori déploient leur logique "par-dessus la tête des intéressés"3, peuvent-elles interférer avec la logique de formation du sens commun propre au "monde de la vie" ? On peut transposer à l'usage des médias le schéma herméneutique de Dilthey : la pratique des médias -en tant qu'expérience singulière- alimente le sens commun, mais, en même temps, c'est dans ce sens commun -le discours des médiasque les individus trouvent les ressources nécessaires pour constituer leurs expériences singulières. Pour mieux comprendre, détaillons ce processus en deux phases :

1 Selon J.-M. Ferry, il convient de dépasser ce schéma, dans la mesure où, dans nos sociétés complexes contemporaines, la formation de l'identité n'est plus liée aux seuls registres discursifs narratif et interprétatif ; il y adjoint l'argumentatif et, surtout, le reconstructif (J.-M. FERRY, Les puissances de l'expérience, Paris, Éditions du Cerf, coll. "Passages", vol. 1, 1991, pp. 101-157 : "Les formes de l'identité").

2 On trouve notamment l'idée d'un "stock de connaissances commun" à tous les individus d'une société donnée chez A. Schütz (voir notamment: N. ZACCAÏREYNERS, Le monde de la vie, vol. 2 : Schütz et Mead, Paris, Éditions du Cerf, coll. "Humanités", 1996.) et chez ses continuateurs, P. BERGER et Th. LUCKMANN (voir : P. BERGER et TH. LUCKMANN, La construction sociale de la réalité (1966), trad., Paris, Armand Colin, 1996). En particulier, on trouve dans la sociologie de ces deux derniers l'idée, déjà présente chez Dilthey, d'une intériorisation de ce qui a été préalablement extériorisé, puis objectivé.

3 N. ZACCAÏ-REYNERS, Le monde de la vie, vol. 3, p. 115 : "On peut expliquer la distinction entre système et monde de la vie en examinant la façon dont s'y effectue la coordination de l'action. Si, au sein du monde vécu, elle demeure orientée normativement, l'intégration systémique d'activités est dépourvue de lien interne avec la validité normative". La présente étude entend directement participer à l'éclaircissement des liens problématiques unissant système (médiatique, en l'occurrence) et monde de la vie. 
- d'une part, en tant que lieu d'une socialisation (les médias en tant que part de l'expérience générale), la confrontation avec les médias pourvoit les individus en "modèles" -cognitifs ou affectifs- à partir desquels (ou en confrontation avec lesquels) ils constituent leurs "expériences singulières";

- en même temps, la pratique médiatique, considérée cette fois comme expérience vécue (les médias en tant qu'intervenant au niveau de l'expérience individuelle), alimente la constitution du sens commun lorsqu'elle se trouve stabilisée dans des récits, puis, lors de la confrontation avec d'autres expériences du même type (la communauté d'interprétation, présente ou imaginaire), ressaisie et interprétée (en famille ou à l'école, par exemple).

En somme, les individus sont mobilisés pour fournir un effort d'interprétation des expériences de vie médiatisées, mais, en même temps, ils trouvent dans les médias eux-mêmes les ressources de sens et de savoir en vue de cet effort ; la pratique médiatique, en tant qu'expérience vécue, alimente la constitution d'un sens commun dont elle se nourrit elle-même en tant que processus de socialisation. Il y a là un risque de décrochage par rapport aux expériences vécues dans la société, risque d'autant plus important que l'exposition aux médias ou leur utilisation est fréquente. En contribuant à créer un sens commun artificiel (du point de vue de son mode d'élaboration), les médias constituent, de notre point de vue, une sorte d'interprétant final ou de Grand Interprétant au sens du sémiologue américain Ch. S. Peirce ${ }^{1}$ : leur intervention dans le double processus décrit ci-dessus limite fortement le type de sens susceptible d'être produit au cours de l'interprétation des messages médiatiques. Cet aspect de ce qu'on pourrait apparenter à une forme de "colonisation du monde vécu par le système" (J. Habermas) laisse entrevoir les limites d'une socialisation par les médias :

1 Pour Peirce, "un signe ou representamen est quelque chose qui tient lieu pour quelque chose sous quelque rapport ou à quelque titre. Il s'adresse à quelqu'un, c'est-à-dire crée dans l'esprit de cette personne un signe équivalent ou peut-être un signe plus développé. Ce signe qu'il crée, je l'appelle l'interprétant du premier signe." (CH. S. PEIRCE, Écrits sur le signe, trad., Paris, Éditions du Seuil, 1978, p. 215, cité in N. ZACCAÏ-REYNERS, op.cit., pp. 50-51). "La signification exacte du représentant résulte d'un procès d'interprétation déployant une chaîne d'interprétants. Le premier interprétant va en effet en appeler un deuxième, qui en appellera lui-même un troisième, etc." (N. ZACCAÏ-REYNERS, op.cit., p. 51). 
la perte de sens commun (...) peut être interprétée dans les termes habermassiens d'une colonisation du monde de la vie et de sa rationalité propre, ancrée dans la pratique du discours ordinaire, par la rationalité systémique et organisationnelle dépourvue d'orientation normative' ${ }^{1}$.

Après avoir envisagé l'"élaboration du sens commun" sous son aspect génétique (comment le sens commun se produit et se reproduitil ?), voyons ce qu'il en est sous son aspect structurel: y a-t-il une spécificité des ressources de sens et de savoir mises à disposition dans le cadre du processus décrit ci-dessus et qui implique les médias ? Il semble en fait que ces ressources soient extrêmement pauvres, tant le discours des médias est fait de spots, clips et autres clichés, soit d'éléments qui se situent dans un registre qu'on pourrait qualifier d' "infranarratif" ou d'"infra-discursif". Ces éléments font partie

Des menaces [qui] pèsent actuellement sur la continuité homogène de ces ressources de sens qui cimentent une communauté en amont de tout discours rationnel. Des menaces de quelle nature ? Elles se manifestent dans la réactivation de registres de discours sur un mode crispé qui instrumentalise les expériences vécues invoquées ou les radicalise (registres religieux, mythique, néo-paganiste, antirationaliste, valorisant l'expressivité...). Mais également par la déchirure ou rupture des strates antérieures de la sémiosis ${ }^{2}$ sociale. Ou encore par la régression sur les niveaux iconiques ou indiciels révélant une mutation de la structure sémiotique de la reproduction culturelle (chute relative de l'écrit, succès des clips et des jeux vidéo, de la bande dessinée... $)^{3}$.

Or, si l'on s'en réfère au schéma de Dilthey, il est impossible pour les individus de s'approprier leur propre expérience individuelle (donc de constituer leur identité) au moyen de ressources de sens commun non discursives, soit sans s'inscrire dans un "récit de vie". Il y aurait dès lors danger d'un déficit de structuration des identités, tant individuelles que collectives (les deux niveaux rétroagissant) ${ }^{4}$.

Ibid., p. 117.

Nous entendons par "sémiosis" le processus d'élaboration du sens.

N. ZACCAÏ-REYNERS, op.cit., p. 116.

4 Cependant, on pourrait nous objecter que le schéma de Dilthey, dépassé en pratique, n'est aujourd'hui plus valide en théorie. En d'autres termes, on pourrait très bien 
Étant donné le rôle essentiel que jouent les ressources discursives dans le processus de socialisation, il est essentiel de faire droit, dans la recherche sur les médias, au discours diffusé. Nous tenons, dans ce cadre, à exprimer notre intérêt pour une forme de discours médiatique en particulier : celui du Journal Télévisé. Sans même nous prononcer quant à la qualité argumentative de ce type de discours ${ }^{1}$, il nous paraît que la narration, en tant que mode de médiation des savoirs (ce qu'elle est aussi chez Dilthey) y est aujourd'hui particulièrement mal menée, car trop souvent dépourvue de cohérence interne, tant au niveau du "récit" que devrait constituer chaque vidéogramme qu'au niveau du "récit" fédérateur et formateur que devrait être le Journal dans son intégralité. Une situation d'autant plus préoccupante que le $J T$ nous paraît constituer le lieu de stabilisation et de construction "objectives" (et non plus intersubjectives) du sens commun (il s'agit, en effet, du moment privilégié au cours duquel l'individu est le plus directement confronté avec l'image que sa communauté se donne prétendument d'elle-même), en même temps que d'"une mise en forme élaborée du sens commun"2. Soit peut-être le lieu privilégié pour la fabrication d'un sens commun artificiel.

penser que les individus élaborent bien quelque chose comme un "sens commun" à partir des ressources infra-discursives ou infra-symboliques (iconiques ou indicielles) mises à leur disposition par les médias. Pour le dire autrement encore : de la sémiosis s'opérant à partir des messages médiatiques ne résulterait pas qu'une dégrammaticalisation (ou perte de sens commun conventionnel), mais aussi une re-grammaticalication fondée sur un sens commun de type nouveau. Mais, même dans ce cas, il n'est pas du tout exclu qu'une telle recherche, en tentant d'élucider les grammaires présidant à la réception, aboutisse à montrer en quoi les styles en question (spots, clips, clichés,...), tant prisés actuellement par le système médiatique, sont précisément ceux qui flattent, en chaque individu, la forme d'identité la plus primitive. "Primitive", au sens non péjoratif d'élémentaire, c'est-à-dire renvoyant à des expériences originelles et constitutives.

1 En effet, pourrait-on déontologiquement concevoir que le $J T$ ait une visée délibérément argumentative?

2 A. MERCIER, Le Journal Télévisé. Politique de l'information et information politique, Paris, Presses de Sciences Po, 1996, p. 268. 
